ARTICLE

DOI: $10.1038 / s 41467-017-01844-2$

\title{
Ultrafast non-radiative dynamics of atomically thin $\mathrm{MoSe}_{2}$
}

Ming-Fu Lin ${ }^{1,2}$, Vidya Kochat ${ }^{3}$, Aravind Krishnamoorthy ${ }^{4}$, Lindsay Bassman ${ }^{4}$, Clemens Weninger $^{1,2}$, Qiang Zheng ${ }^{5}$, Xiang Zhang ${ }^{3}$, Amey Apte ${ }^{3}$, Chandra Sekhar Tiwary ${ }^{3}$, Xiaozhe Shen ${ }^{5}$, Renkai Li ${ }^{5}$, Rajiv Kalia ${ }^{4}$, Pulickel Ajayan ${ }^{3}$, Aiichiro Nakano ${ }^{4}$, Priya Vashishta ${ }^{4}$, Fuyuki Shimojo ${ }^{6}$, Xijie Wang ${ }^{5}$, David M. Fritz ${ }^{1} \&$ Uwe Bergmann (10 ${ }^{2}$

Photo-induced non-radiative energy dissipation is a potential pathway to induce structuralphase transitions in two-dimensional materials. For advancing this field, a quantitative understanding of real-time atomic motion and lattice temperature is required. However, this understanding has been incomplete due to a lack of suitable experimental techniques. Here, we use ultrafast electron diffraction to directly probe the subpicosecond conversion of photoenergy to lattice vibrations in a model bilayered semiconductor, molybdenum diselenide. We find that when creating a high charge carrier density, the energy is efficiently transferred to the lattice within one picosecond. First-principles nonadiabatic quantum molecular dynamics simulations reproduce the observed ultrafast increase in lattice temperature and the corresponding conversion of photoenergy to lattice vibrations. Nonadiabatic quantum simulations further suggest that a softening of vibrational modes in the excited state is involved in efficient and rapid energy transfer between the electronic system and the lattice.

\footnotetext{
${ }^{1}$ Linac Coherent Light Source, SLAC National Accelerator Laboratory, Menlo Park, CA 94025, USA. ${ }^{2}$ Stanford PULSE Institute, SLAC National Accelerator Laboratory, Menlo Park, CA 94025, USA. ${ }^{3}$ Department of Materials Science and NanoEngineering, Rice University, Houston, TX 77005, USA.

${ }^{4}$ Collaboratory for Advanced Computing and Simulations, Department of Physics \& Astronomy, Department of Computer Science, Department of Chemical Engineering \& Materials Science, Department of Biological Sciences, University of Southern California, Los Angeles, CA 90089-0242, USA. ${ }^{5}$ SLAC National Accelerator Laboratory, Menlo Park, CA 94025, USA. ${ }^{6}$ Department of Physics, Kumamoto University, Kumamoto 860-8555, Japan. Correspondence and requests for materials should be addressed to U.B. (email: bergmann@slac.stanford.edu)
} 
E nergy loss pathways are important considerations for the design of optoelectronic devices based on nanomaterials like transition metal dichalcogenides (TMDCs) $)^{1,2}$, which are promising candidates for ultrafast photodetection ${ }^{3}$, valleytronics ${ }^{4}$, and field effect transistors ${ }^{5,6}$. Much of the recent attention has been focused on the radiative relaxation and defect-mediated non-radiative bimolecular recombination of excitons, which have been shown to drastically limit photoluminescence quantum yield even at the monolayer limit $(\sim 0.4 \%)^{7-12}$. In addition, considerable effort has been expended in the strain engineering ${ }^{13,14}$ and in identifying strategies for chemical passivation of defects to improve light emission efficiency and device performance ${ }^{15,16}$. The nonradiative channel is the dominant energy loss pathway in defect-free monolayers at high charge carrier densities but is less well explored ${ }^{1,6,9,15}$. Recent work from Ruppert et al. ${ }^{17}$ has shown that an optically excited monolayer $\mathrm{WS}_{2}$ relaxes nonradiatively to lattice vibration, causing the absorption edge to redshift due to a small temperature jump of $\sim 40 \mathrm{~K}$. This nonradiative process is promising for triggering light-driven atomic motion required for structural-phase transition, but so far, it is not well understood due to a lack of experimental methods to quantify atomic motion and lattice temperature directly.

In this study, we utilize mega-electronvolt ultrafast electron diffraction (MeV-UED $)^{18}$ as a probe to measure mean-square atomic displacements and the corresponding local temperature in bilayer $\mathrm{MoSe}_{2}$ samples photoexcited to a high carrier density $\left(\sim 10^{14} \mathrm{~cm}^{-2}\right)$, which lies in the electron-hole plasma regime. At this high charge carrier concentration, the laser fluence of the optical pump is high enough to deposit the energy required for initiating a possible structural-phase transition (temperature jump of $\sim 1000-\mathrm{K}$ and $\sim 0.3-\AA$ difference of lattice constant between $2-\mathrm{H}$ and $1-\mathrm{T}$ phases). Time-resolved electron diffraction allows us to directly explore laser-induced atomic motion and structural disorder with $\sim 200$-fs temporal resolution, and thus to directly quantify the efficiency of nonradiative energy channels in these two-dimensional materials. We monitor the dependence of the resulting lattice disorder on the photoinjected carrier concentration by varying the pump fluence at two different wavelengths. The observed subpicosecond increase in structural disorder and lattice temperature is consistent with ultrafast conversion of electronic energy to atomic motion via softened phonon modes observed in our first-principles density functional theory (DFT) and nonadiabatic quantum molecular dynamics (NAQMD) simulations. Furthermore, the observed ultrafast temperature rise is linearly proportional to the absorption of the pump energy calibrated to a saturable absorber model, indicating a high efficiency of the nonradiative decay channel.

\section{Results}

Time-resolved electron diffraction. A schematic of the experimental procedure and a representative electron diffraction pattern and intensity profile is displayed in Fig. 1. Each diffraction pattern is generated by the accumulation of over $\sim 7000$ pulses with effective charge of $\sim 20 \mathrm{fC}$ per pulse at the sample target. Several families of diffraction planes in the reciprocal lattice are observed. All the pump-probe traces at a given reciprocal lattice vector $\mathbf{Q}$ (i.e., $2 \pi / d$ ) are averaged over six equivalent planes. Diffraction intensity for a lattice plane is calculated from the full diffraction pattern by the integration of photodetector counts on a small region around the corresponding reciprocal lattice vector. Negative delay times are shown when the electron probe beam precedes the optical pump beam on the sample target. A red kinetic plot in Fig. 1 displays a representative temporal profile of the measured diffraction intensity, which shows a rapid exponential decay of the initial diffraction intensity value $I_{0}$ to a new lower value of $I$ at a positive delay time due to the increased incoherent atomic motion induced by the optical pumping. The ratio of these two intensities allows us to estimate the atomic

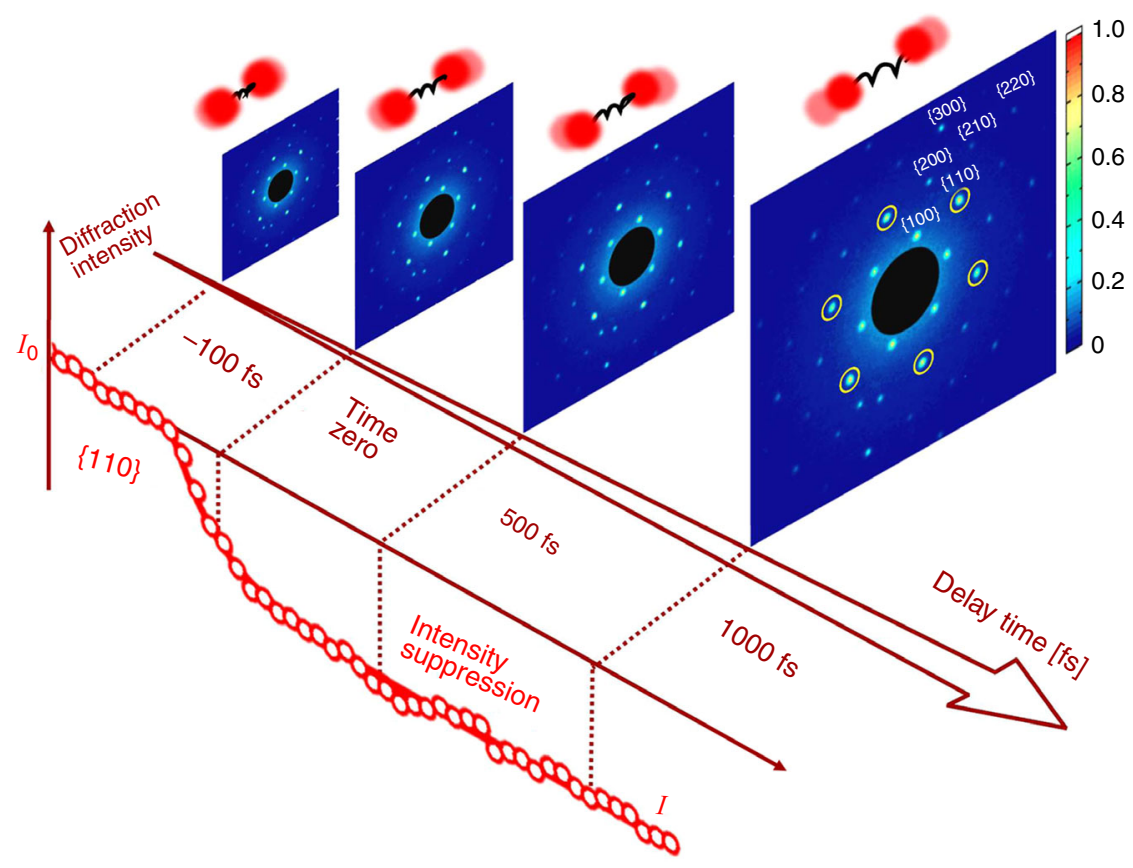

Fig. 1 Time-resolved mega-eV electron diffraction. Snapshots of electron diffraction of $\mathrm{MoSe}_{2}$ bilayer for Debye-Waller factor (DWF) measurements. Several families of diffraction planes are labeled in the last diffraction image for clarity (i.e., $\{100\},\{110\},\{200\},\{210\},\{300\}$, and $\{220\}$ ). A timeresolved kinetic plot that illustrates the suppression of diffraction intensity at $\{110\}$ family is shown, where $I_{0}$ and $I$ denote the Bragg peak diffraction intensities at negative and positive delay times from the fit of experimental results. Each image is accumulated over 7000 pulses from multiple scans with effective charge of $\sim 20 \mathrm{fC}$ per pulse at the sample target. The mean-square displacements, $\left\langle\Delta u^{2}\right\rangle_{\Delta T}$, are obtained by plotting $\ln \left(\frac{I_{0}}{T}\right)$ vs. $Q^{2}$, where $Q$ is $2 \pi$ over interplanar spacing $d$ 
a

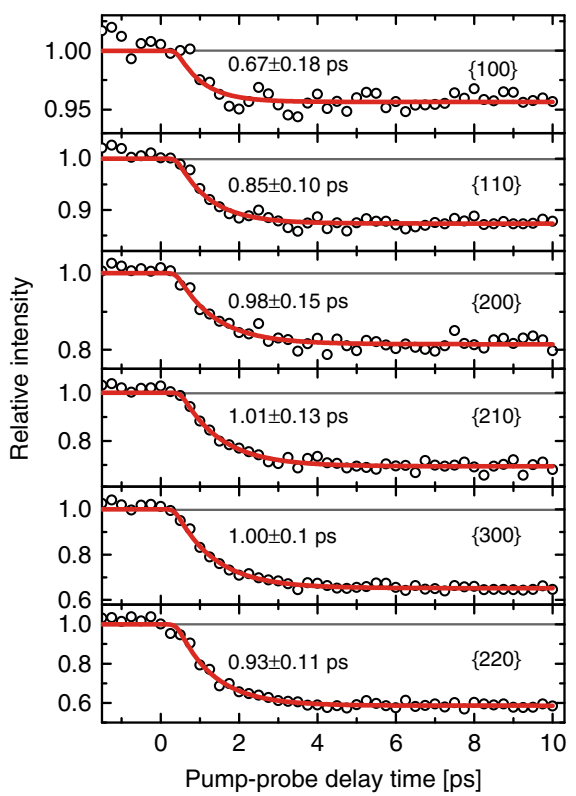

b

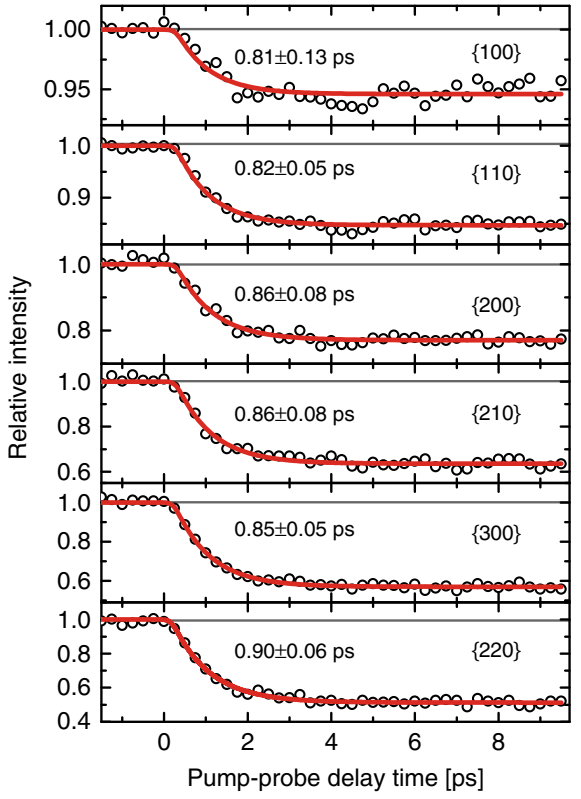

C
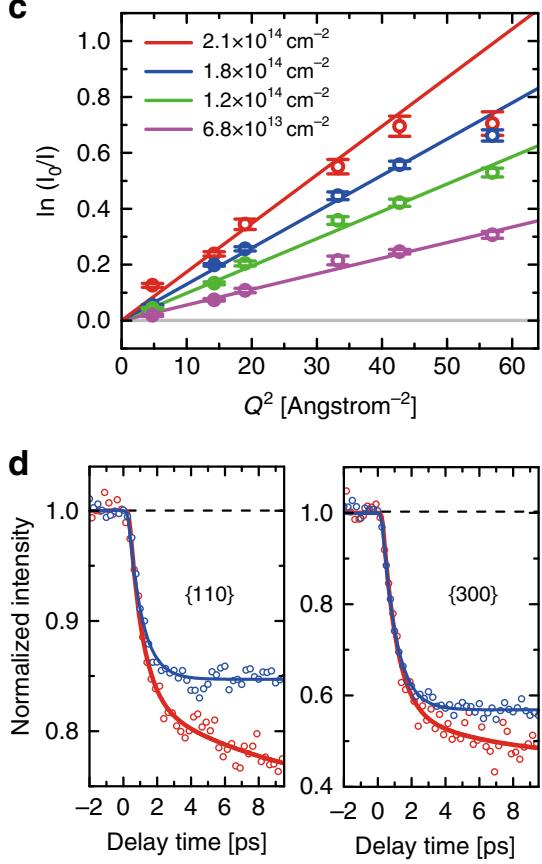

Fig. 2 Pump-probe kinetics and Debye-Waller responses at 400-nm photoexcitation. Pump-probe kinetic plots for six diffraction planes at a carrier density of (a) $1.2 \pm 0.1 \times 10^{14} \mathrm{~cm}^{-2}$ and (b) $1.8 \pm 0.1 \times 10^{14} \mathrm{~cm}^{-2}$ at $400 \mathrm{~nm}$ excitation for the $\mathrm{MoSe}_{2}$ bilayer, and (c) Debye-Waller responses at four carrier densities. A large deviation between linear fit and experimental data occurs at the highest carrier density (red). At this density, the first five data points are used for the linear fit. The corresponding temperatures calculated from the slopes are presented in the context and Supplementary Information. Error bars represent $68 \%$ confidence interval and are calculated from the propagation of standard error of the mean from the fit of averaged multiple pump-probe scans. d Kinetic plots for $\{110\}$ and $\{300\}$ planes at carrier density at $1.8 \pm 0.1 \times 10^{14} \mathrm{~cm}^{-2}$ (blue) and $2.1 \pm 0.1 \times 10^{14} \mathrm{~cm}^{-2}$ (red), respectively, for comparison. A complex Bragg peak intensity decay occurs at the highest carrier density (red), which may suggest an energy redistribution between different phonons from a strong carrier-modified potential energy surface

disorder and the resulting temperature jump produced by the optical excitation, which will be further discussed later in this paper.

Theoretical approach. Our UED experiments are further supported by NAQMD simulations on $\mathrm{MoSe}_{2}$ bilayer crystals, which follow the trajectories of all atoms while computing interatomic forces quantum mechanically from first principles ${ }^{19}$, including the dynamics of electrons and nuclei ${ }^{20}$. Electronic excitations are described within the framework of linear-response time-dependent DFT, which is a proven method to investigate ultrafast nonradiative relaxation in other electronically excited material systems $^{21}$. In this study, nonadiabatic transitions between electronic excited states assisted by molecular motions are treated with a surface-hopping approach.

In order to elucidate atomic dynamics observed in our QMD simulations, we also calculated phonon dispersion curves for the ground-state and electronically excited $\mathrm{MoSe}_{2}$ bilayers using DFT and the $\Delta$-SCF method. Specifically, for excited electron-hole concentration of $1.2 \times 10^{14} \mathrm{~cm}^{-2}\left(2.4 \times 10^{14} \mathrm{~cm}^{-2}\right)$ used in this study, we promote one (two) electrons from the valence band to the conduction band. The comparison of experimental results from $\mathrm{Si}_{3} \mathrm{~N}_{4}$-supported $\mathrm{MoSe}_{2}$ flakes to simulation results from suspended $\mathrm{MoSe}_{2}$ bilayers is justified for two reasons. First, $\mathrm{MoSe}_{2}$ bilayer forms a type-I heterojunction with the insulating $\mathrm{Si}_{3} \mathrm{~N}_{4}$ substrate ( $5-\mathrm{eV}$ bandgap), which precludes a charge transfer process. Second, Raman microscopy and photoluminescence measurements from the $\mathrm{Si}_{3} \mathrm{~N}_{4}$-supported $\mathrm{MoSe}_{2}$ agree with previous measurements on different substrates ${ }^{22}$, indicating a lack of substrate effect on $\mathrm{MoSe}_{2}$ vibration modes.
Debye-Waller response of bilayer $\mathrm{MoSe}_{2}$. Figure 2 summarizes our experimental results for bilayer $\mathrm{MoSe}_{2}$ samples photoexcited at $400 \mathrm{~nm}$. The temporal evolution of the measured diffraction intensities for six diffraction families at carrier densities of $1.2 \pm$ $0.1 \times 10^{14} \mathrm{~cm}^{-2}$ and $1.8 \pm 0.1 \times 10^{14} \mathrm{~cm}^{-2}$ is shown in Fig. $2(\mathrm{a}, \mathrm{b})$, respectively. Note that all the pump-probe kinetics are normalized to one at the negative delay time $\left(I_{0}\right)$, so that the fractional drop of intensity can be quickly identified for different diffraction families. Each pump-probe trace is then fit to an exponential function convoluted with an 200 -fs full-width at half-maximum instrument response function (time resolution) estimated for the $\mathrm{MeV}$-UED system. Thus, the obtained $I$ and $I_{0}$ from the fit are the relative electron diffraction intensities at far positive and negative delay times, respectively, as shown in the red curves in Fig. 2a, b. The kinetic plot at a lower carrier density of $0.68 \pm 0.1 \times 10^{14} \mathrm{~cm}$ -2 is presented in Supplementary Fig. 2a in Supplementary Note 3 . The standard deviation error bars are approximately 0.04 by using multiple scans with a total of $\sim 7000$ pulses per delay time point. The primary observation in the intensity profiles is the ultrafast subpicosecond decay of diffraction intensities for all families of planes considered in our study. This is approximately a factor two faster than the time constant reported for the monolayer $\mathrm{MoS}_{2}$ at a similar carrier density $(\tau \sim 1.7 \mathrm{ps})^{23}$. Furthermore, the invariant steady-state diffraction intensity up to ten picoseconds indicates that the lattice is thermalized for delay times longer than one picosecond after optical excitation. The experimental time constants do not show any obvious changes as a function of the carrier density produced at $400 \mathrm{~nm}$ and $800 \mathrm{~nm}$, implying a minor role of high-order carrier kinetics in these carrier density regimes.

Below, we consider three possible mechanisms to explain the ultrafast appearance of lattice disorder. First, the lattice energy 

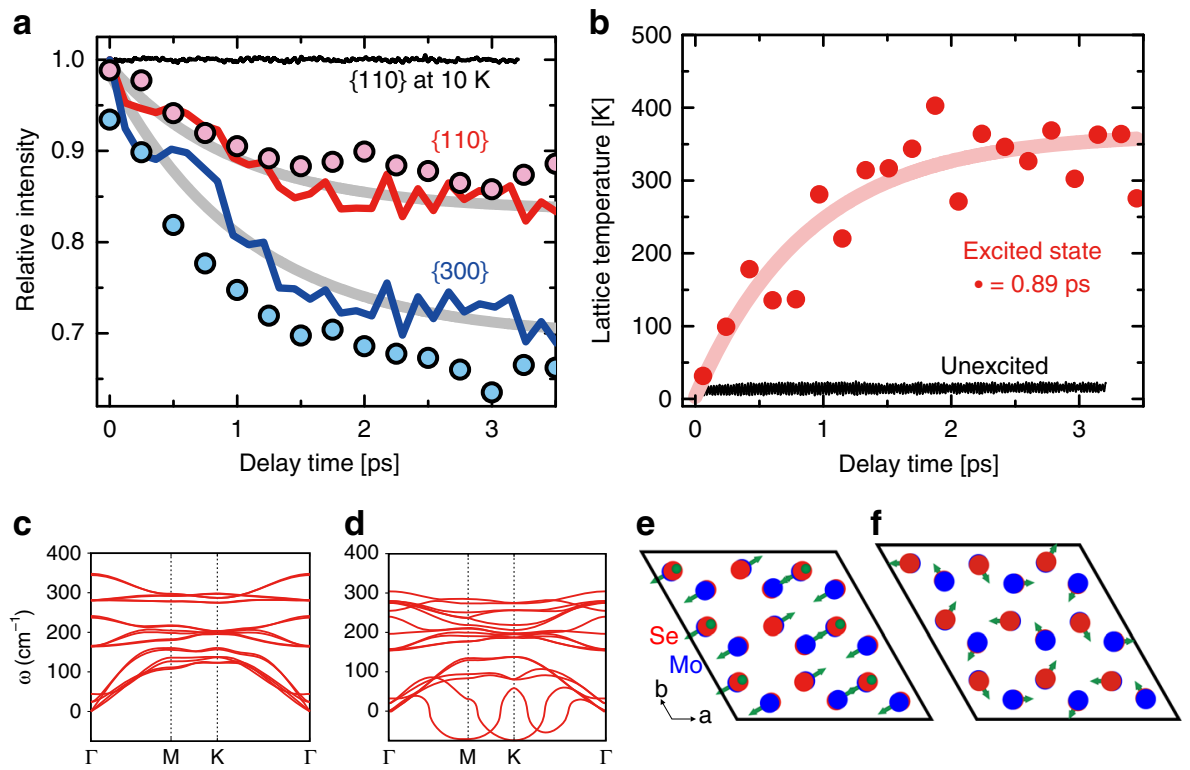

Fig. 3 NAQMD simulations of bilayer MoSe 2 a Debye-Waller factor for the MoSe $e_{2}$ bilayer as a function of delay time for the $\{110\}$ and $\{300\}$ planes calculated from NAQMD simulations at carrier density of $0 \mathrm{~cm}^{-2}$ (black) and $1.2 \times 10^{14} \mathrm{~cm}^{-2}$ (red and blue lines). Photoexcitation results in a marked increase in structural disorder and a reduction in the Bragg peak intensity. Exponential fits to the simulations yield a time constant of $\sim 1 \mathrm{ps}$, as shown in the gray curves. The experimental results shown in circles are the bilayer $\mathrm{MoSe}_{2}$ at a carrier density of $1.2 \times 10^{14} \mathrm{~cm}^{-2}$. Note that there are no normalization factors between the experimental and simulation results. $\mathbf{b}$ Instantaneous temperature in the NAQMD simulation cell due to photoexcitation at time $t=$ 0 shows a transient temperature increase from $10 \mathrm{~K}$ to $300 \mathrm{~K}$ over $1.5 \mathrm{ps}$. c, $\mathbf{d}$ display phonon dispersion curves for the MoSe $\mathrm{S}_{2}$ bilayer at the photoexcited carrier density of $\mathbf{c} 0 \mathrm{~cm}^{-2}$, (d) $1.2 \times 10^{14} \mathrm{~cm}^{-2}$, respectively. e, f Eigenvectors for the soft acoustic modes at the M-point and K-point, respectively

could result from the high-order kinetics such as nonradiative bimolecular and Auger recombination through annihilation of photoexcited excitons. The obtained carrier density for this experiment is $\sim 10^{14} \mathrm{~cm}^{-2}$ such that an electron-hole plasma is produced from screened exciton dissociation (i.e., the Mott density is $\left.\sim 10^{14} \mathrm{~cm}^{-2}\right)^{24-27}$. It has been reported that the bimolecular exciton-exciton annihilation or Auger process mediated by defects/traps occurs efficiently in $\mathrm{MoSe}_{2}$ and $\mathrm{MoS}_{2}{ }^{7,8,28}$. However, this happens at exciton densities below $\sim 10^{13} \mathrm{~cm}^{-2}$ allowing intact excitons to annihilate each other in the time scale of a few picoseconds. At a higher charge carrier density $\left(\sim 10^{14} \mathrm{~cm}^{-2}\right)$, excitons dissociate into free carriers due to the increased screening of Coulomb interaction in the bound charges $^{24,27}$. Therefore, the exciton-exciton annihilation may not be able to account for this fast appearance of lattice disorder. Our power dependence measurement at $400 \mathrm{~nm}$ does not show clear changes of time constants as a function of carrier density in the electron-hole plasma regime. This suggests that the high-order carrier kinetics such as Auger processes does not play an important role in our experiments.

The second possible explanation for the rapid decay of diffraction intensity may be due to the induction of nonthermal melting. Figure 2c shows the logarithmic ratios of measured diffraction intensities vs. the diffraction families in the squarereciprocal space at four carrier densities (i.e., $\ln \left(I_{0} / I\right)$ vs. $Q^{2}$ ). The effective mean-square displacements associated with different carrier concentrations can be estimated from the slope ${ }^{29,30}$, $\Delta\left\langle u^{2}\right\rangle_{\Delta T}$, by using the Debye-Waller equation as shown in Eqs. (1) and (2).

$$
\begin{gathered}
I=I_{0} e^{-\Delta\left\langle u^{2}\right\rangle_{\Delta T} \times Q^{2}} \\
\ln \left(I_{0} / I\right)=\Delta\left\langle u^{2}\right\rangle_{\Delta T} \times Q^{2}
\end{gathered}
$$

The calculated mean-square displacements for the four different carrier concentrations are $5.6( \pm 0.2) \times 10^{-3} \AA^{2}, 9.8$
$( \pm 0.3) \times 10^{-3} \AA^{2}, 13.0( \pm 0.4) \times 10^{-3} \AA^{2}$, and $17.4( \pm 1.2) \times 10^{-3} \AA^{2}$ respectively, corresponding to a root-mean-square atomic displacement, $\sqrt{\Delta\left\langle u^{2}\right\rangle_{\Delta T}}$, of $0.075 \pm 0.001 \AA, 0.099 \pm 0.002 \AA$, $0.114 \pm 0.002 \AA$, and $0.132 \pm 0.005 \AA$. These photoinduced atomic displacements range from $2 \%$ to $5 \%$ of the in-plane lattice constant of $3.3 \AA^{31}$, which is below the Lindemann criterion for a solid-to-liquid phase transition (i.e., $\sim 10 \%-20 \%)^{32}$. In addition, these displacements are much less than what would be expected for highly inertial atomic motion initiated by an intense optical excitation (highly nonthermal melting) ${ }^{33-35}$. We estimate a $\left(3 k_{\mathrm{B}} T / m\right)^{1 / 2}=2.9 \AA$ per picosecond mean atomic velocity for $\mathrm{MoSe}_{2}$ at room temperature, where $k_{\mathrm{B}}, m$, and $T$ are the Boltzmann constant, averaged mass, and room temperature, respectively. At this velocity, the mean atomic displacements would be $1.45 \AA$ at a 500 -fs time delay, corresponding to $\sim 44 \%$ of in-plane lattice constant. This is $\sim 10$ times higher than our observations at the highest pump fluence. Thus, our data are not consistent with a nonthermal melting and strong atomic inertial processes from potential deformation by the strong laser field.

Another possibility is the existence of strong electron-phonon coupling, which has been postulated for molybdenum chalcogenides with dynamics occuring on a subpicosecond time scale ${ }^{36}$. Figure $2 \mathrm{~d}$ presents a comparison of kinetic plots for $\{110\}$ and $\{300\}$ planes at two carrier densities (i.e., $1.8 \times 10^{14} \mathrm{~cm}^{-2}$ (blue) and $\left.2.1 \times 10^{14} \mathrm{~cm}^{-2}(\mathrm{red})\right)$. For the lower carrier density, a fast subpicosecond single-exponential decay of the diffraction intensity is observed. For the higher carrier density, we see an additional slower decay with a lifetime of $\sim 10$ ps in addition to the fast decay. Such a complex decay of diffraction intensity was previously reported for another layered material, graphite, where it was attributed to the coexistence of electron-phonon and phonon-phonon scattering, as well as to electron-phonon coupling to two different vibration modes ${ }^{29,30}$. In our experiments, the observed crossover from single exponential to biexponential intensity decay with increasing excited charge carrier concentration (and correspondingly different potential 

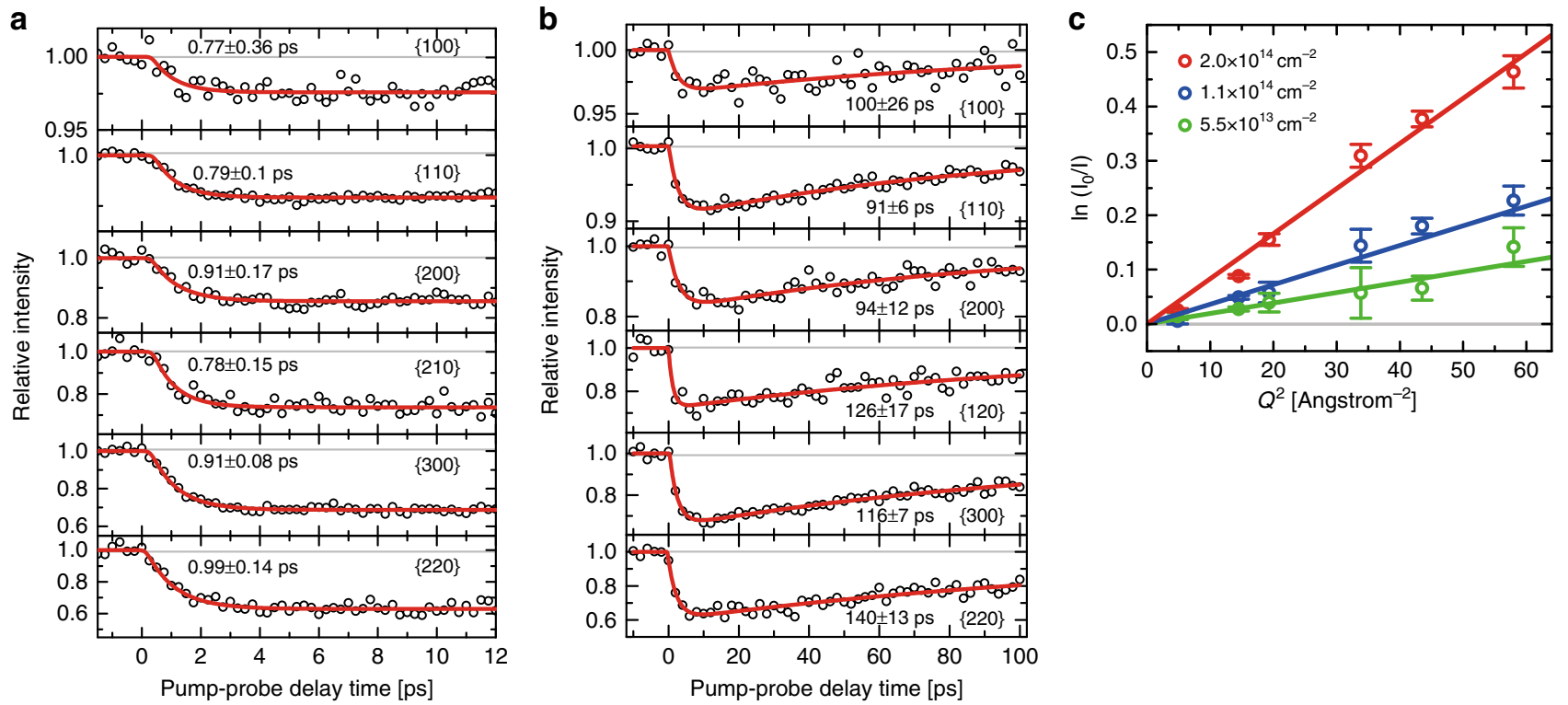

Fig. 4 Pump-probe kinetics and Debye-Waller responses at $800 \mathrm{~nm}$ photoexcitation. a Time-resolved kinetic plots of MoSe 2 bilayer for six diffraction families, as depicted in the parenthesis at a carrier density of $2.0 \pm 0.2 \times 10^{14} \mathrm{~cm}^{-2}$ excited at $800 \mathrm{~nm}$. The resultant subpicosecond time constants are consistent with measurements at $400 \mathrm{~nm}$. b At the same carrier density and wavelength for the bilayer with the pump-probe scan up to 100 ps. A clear recovery of Bragg peak intensity is shown ( $100 \mathrm{ps}$ ). This is due to the heat propagation from the bilayer MoSe $\mathrm{e}_{2}$ to the substrate. c Power-dependent Debye-Waller responses of a bilayer for three effective carrier densities at $800 \mathrm{~nm}$ photoexcitation. Error bars represent $68 \%$ confidence interval and are obtained from the propagation of standard error of the mean from the fit of averaged multiple pump-probe scans. The mean-square displacements obtained from the linear least-square fit of data are shown in Supplementary Table 3. Note that at the highest carrier density, the Debye-Waller response is still linear without a large deviation, as seen in Fig. 2c

energy surfaces) is indicative of electron-phonon coupling to one or more decay channels at low and high charge carrier densities, respectively. The other diffraction planes also reveal two contributions from the fast and the slow decay channels, and the results are presented in Supplementary Fig. $2 \mathrm{~b}$.

NAQMD simulation results. To interpret this ultrafast subpicosecond change of Bragg peak intensity as a function of delay time, we performed NAQMD simulations of $\mathrm{MoSe}_{2}$ bilayer in the electronically ground and excited states, respectively. Figure 3a shows the Debye-Waller factors (DWF) for the $\{110\}$ (red) and $\{300\}$ (blue) families of planes calculated from an ensemble of four photoexcited NAQMD atomic trajectories and compared to the Debye-Waller factors for the unexcited $\mathrm{MoSe}_{2}$ bilayer at $10 \mathrm{~K}$. Single-exponential decay fits to the $\{110\}$ and $\{300\}$ planes show an ultrafast increase in structural disorder with time constants of $\sim 1$ ps (gray), and magnitude of DWF decay $(\Delta=0.15$ and 0.3 ) that are qualitatively consistent with experimental measurements (circles). This subpicosecond dynamics is further supported by Fig. 3b, which shows a plot of the instantaneous temperature of atoms in the simulation cell as a function of time. The calculated temperature of the simulation cell increases from the initial value of $10 \mathrm{~K}$ to a steady-state value of $\sim 350 \mathrm{~K}$ in a lifetime scale of $\sim 0.89$ ps. Snapshots of time-resolved atomic velocity distributions at two delay times are presented in Supplementary Fig. 3 and Supplementary Note 4 to rationalize a fast equilibration of lattice temperature in our experiments.

To further understand the underlying ultrafast atomistic mechanisms, we calculated phonon dispersion curves as a function of electronic excitation with the method described previously for the $\mathrm{MoSe}_{2}$ bilayer. This allows us to study the effect of excited charge carrier population on the potential energy surface of the crystal. Figure $3 \mathrm{c}, \mathrm{d}$ shows the phonon dispersion of the $\mathrm{MoSe}_{2}$ bilayer in the ground and excited states, respectively. Electronic excitation causes two primary differences to the potential energy surface of the crystal. First, the frequencies of atomic vibrations decrease uniformly throughout the Brillouin zone with increasing excited charge carrier concentration, reflecting a weakening of interatomic bonds leading to greater atomic displacements. Second, with increased charge carrier concentration, softening of zone-edge phonons is observed at the $\mathrm{M}(0.5,0,0)$ and $\mathrm{K}(0.33,0.33,0)$ points in the Brillouin zone. Eigenvectors corresponding to these soft modes are shown in Fig. $3 \mathrm{e}, \mathrm{f}$, respectively. The flattening of the potential energy surface corresponding to the soft vibration mode at the M-point enables in-plane displacement of Mo ions to form Mo-Mo dimer chains and is particularly important for displacements corresponding to the $2 \mathrm{H} \rightarrow 1-\mathrm{T}^{\prime}$ phase transition of $\mathrm{MoTe}_{2}{ }^{37,38}$. Such soft modes represent an instability in the $2-\mathrm{H}$ crystal structure, but do not necessarily imply a spontaneous structural transformation from the $2 \mathrm{H}$ to the 1-T' crystal structure, which would require, in addition, barrierless motion of Mo and Se atoms over $0.3 \AA$ from the $2 \mathrm{H}$ to the $1-\mathrm{T}$ ' lattice positions. Further, the largest observed RMS displacement in our pump-probe experiments is $0.13 \AA$ prior to the onset of irreversible sample damage, which is significantly smaller than $0.3 \AA$ required for a structural-phase transformation. However, the existence of such softened vibration modes induced by carrier population is an indication of strong electron-phonon coupling which could enable efficient relaxation of electronic energy to the lattice ${ }^{39,40}$. Phonon softening at $\mathrm{M}$ and $\mathrm{K}$ points in electron-doped $\mathrm{MoS}_{2}$ crystals was accompanied by large electron-phonon coupling constants at these $q$-points ${ }^{41}$. Similarly, in materials like amorphous bismuth and gallium, the large range of motion enabled by the soft vibrations gives rise to anomalously high electron-phonon coupling constants ${ }^{42}$.

Debye-Waller responses at $800-\mathbf{n m}$ photoexcitation. This strong electron-phonon coupling enables a very efficient relaxation of excited carrier energy to the lattice disorder, and this suggests that in the electron-hole plasma regime, the transient 

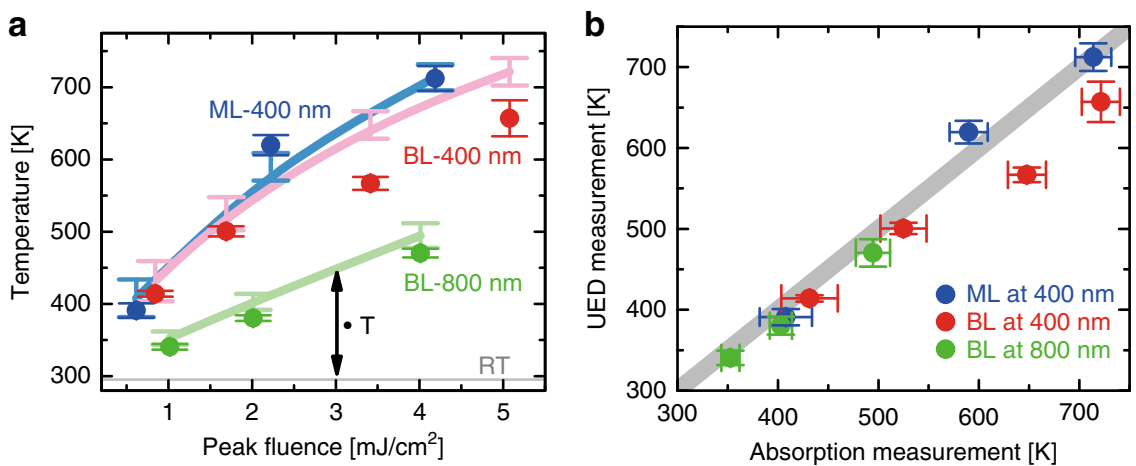

Fig. 5 Temperature correlation plots for atomically thin $\mathrm{MoSe}_{2}$. a Temperatures of atomic thin MoSe $e_{2}$ from UED results (solid circles) and from effective absorption calculated from a saturable absorber model (solid lines). $\Delta T$ denotes the temperature jump above room temperature (RT). ML and BL symbolize the monolayer and bilayer, respectively. Error bars in UED results denote $68 \%$ confidence interval and are obtained from propagation of standard error of the mean from the fit of averaged multiple pump-probe scans. b Correlation plot between UED results and estimates from effective absorption. The gray solid line denotes a full linear correlation, implying a unity of nonradiative quantum yield to lattice disorder. Horizontal error bars in the absorption measurements are calculated by taking into account the uncertainties of powermeter measurements and saturation intensities in ref. 48

lattice temperature rise is simply proportional to the absorbed pump energy. In order to understand the impact of excited charge carrier energy on lattice dynamics, we compare the measured lattice disorder produced by the optical pumping at $400 \mathrm{~nm}$ to results by $800-\mathrm{nm}$ photoexcitation. In particular, at $800-\mathrm{nm}$ excitation, the photon energy is half of a $400-\mathrm{nm}$ photon. This allows us to produce $\sim 50 \%$ of lattice disorder and temperature jump in comparison to $400-\mathrm{nm}$ photoexcitation at a similar carrier density. Indeed, from our experiments at $800 \mathrm{~nm}$, we observed a strong laser-driven Bragg peak intensity decay implying that the lattice disorder results from the electron-hole plasma-driven and phonon-softening nonradiative process. The kinetics at two different pump-probe delay ranges at $800-\mathrm{nm}$ photoexcitation are shown in Fig. 4a, b, respectively. We chose similar carrier densities at $800 \mathrm{~nm}$ compared to $400-\mathrm{nm}$ experiments. In Fig. 4a, the resulting fast time constants are on subpicosecond time scales without any apparent $Q^{2}$ and carrier density dependence, consistent with the observation at $400-\mathrm{nm}$ photoexcitation. This implies that the nonthermal melting and high-order carrier kinetics do not play an important role at $800-\mathrm{nm}$ photoexcitation in this carrier density regime. Note that in our experiments, the irreversible sample damage from the accumulation of quasi-stationary heating does not exist because the heat propagation to the PMMA and silicon nitride substrate occurs on a time scale of 100 ps, as shown in Fig. $4 b^{23}$, which is eight orders of magnitude shorter than the time between electron pulses (i.e., 1/180 s). This ensures that the sample target equilibrates to room temperature before the next pump-probe cycle. This is very different from a recent experiment using Raman microscopy equipped with a CW and high-repetition-rate lasers at a diffraction-limit focus $\left(\sim 1 \mu \mathrm{m}^{2}\right)^{43}$. In that experiment, the average pump power was much higher than in the experiment presented here (i.e., $1000 \mathrm{~W}$ per $\mathrm{cm}^{2}$ vs. $1 \mathrm{~W}$ per $\mathrm{cm}^{2}$ ), but the peak power on the other hand is much lower. This indicates that the Raman experiments are mostly susceptible to the damage from the continuous heating, while for higher peak power, the damage results from single-shot ablation. Figure $4 \mathrm{c}$ shows the Debye-Waller responses as a function of carrier density at $800-\mathrm{nm}$ photoexcitation, which allows us to measure meansquare displacements of a bilayer, as described previously at $400-\mathrm{nm}$ photoexcitation.

Temperature jump from UED measurements and correlation plots. We have also performed power dependence measurements and NAQMD simulations of monolayer $\mathrm{MoSe}_{2}$ samples, as displayed in Supplementary Figs. 4 and 5 in Supplementary Notes 5 and 6, respectively. We observed qualitatively similar time constants, Debye-Waller responses, and mean-square displacements as seen in the bilayer case. A comparison of experimental results between the monolayer and bilayer is shown in Supplementary Table 3 and Supplementary Note 2. In order to calculate the temperature jump of $\mathrm{MoSe}_{2}$ from the measured mean-square displacement, we apply an equation that employs Debye model with assumptions of harmonic approximation and isotropic vibration distribution with a cutoff frequency for the estimation of density of state ${ }^{44}$. This allows us to condense the integration of various phonon modes down to a simple analytical equation, as shown in Eqs. (3)-(5)

$$
\begin{gathered}
\Delta\left\langle u^{2}\right\rangle_{\Delta T}=\left\langle u^{2}\right\rangle_{T}-\left\langle u^{2}\right\rangle_{298 \mathrm{~K}} \\
\left\langle u^{2}\right\rangle_{T}=\frac{3 \hbar^{2}}{m k_{\mathrm{B}} \theta_{\mathrm{D}}}\left[\left(\frac{T}{\theta_{\mathrm{D}}}\right)^{2} P_{\left(\theta_{\mathrm{D}} / T\right)}+\frac{1}{4}\right] \\
P_{\left(\theta_{\mathrm{D}} / T\right)}=\int_{0}^{\frac{\theta_{\mathrm{D}}^{T}}{T}} \frac{x}{e^{x}-1} \mathrm{~d} x \cong 1.6449\left(1-e^{-0.64486 \frac{\theta_{\mathrm{D}}}{T}}\right),
\end{gathered}
$$

where $\hbar, m, k_{\mathrm{B}}$, and $\theta_{\mathrm{D}}$ are the reduced Planck constant, averaged molecular mass, Boltzmann constant, and Debye temperature of the crystal, respectively. Here, we use $84.63 \mathrm{~g}$ per mole and $193 \mathrm{~K}$ for the averaged molar mass and Debye temperature, respectively ${ }^{45}$. Equation (4), with function $P_{\left(\theta_{\mathrm{D}} / T\right)}$, can be solved analytically from the known Debye temperature, as shown in Eq. $(5)^{46}$. The efficiency of optical energy conversion into the thermal motion of a lattice is estimated by a comparison of temperature jump measured from the UED experiments to temperature increase calculated using absorption of the pump beam, the specific heat of the $\mathrm{MoSe}_{2}(\mathrm{~S}=0.278 \mathrm{~J} \text { per } \mathrm{g} \text { per } \mathrm{K})^{47}$, sample density, and volume. Detailed estimation of the temperature jumps from the absorption is presented in Supplementary Note 1 . The calculated temperature jumps in a bilayer using Eqs. (3)-(5) are $116 \pm 4 \mathrm{~K}, 203 \pm 7 \mathrm{~K}, 269 \pm 9 \mathrm{~K}$, and $359 \pm 25 \mathrm{~K}$, respectively, corresponding to the temperatures of $414 \pm 4 \mathrm{~K}, 501$ $\pm 7 \mathrm{~K}, 567 \pm 9 \mathrm{~K}$, and $657 \pm 25 \mathrm{~K}$ at four carrier densities at 400-nm optical excitation. At similar carrier densities, optical excitation by the 400-nm pump results in a larger transient lattice temperature jump than an $800-\mathrm{nm}$ pump. The measured and estimated temperatures from UED and absorption, respectively, are shown in the following section. This correlation between UED 
results and effective absorption allows us to directly quantify the efficiency of the nonradiative channel.

Figure 5a displays UED-measured temperatures (i.e., solid circles) as a function of pump fluence. A saturable absorber model of $\mathrm{MoSe}_{2}$ is used to calculate the effective absorption ${ }^{48}$, and the corresponding temperatures (i.e., solid lines) are plotted with the UED results (circles). We note that the reported saturation intensities $\left(I_{\mathrm{s}}\right)$ from Wang et al. ${ }^{48}$ are used. The predicted temperature jumps from the saturated absorption model agree qualitatively with our UED measurements. We observe a slightly faster temperature rise for the monolayer (ML) than for the bilayer (BL). This can be rationalized as an absorption difference per unit layer between the monolayer and bilayer. In addition, photoexciting the bilayer at $800 \mathrm{~nm}$ reveals roughly half the temperature jump in comparison to the bilayer at $400 \mathrm{~nm}$. This is a result of half the energy per $800-\mathrm{nm}$ photon compared to that absorbed per $400-\mathrm{nm}$ photon.

Figure 5b displays a correlation plot between temperature changes from UED results and from estimated absorption. The experimental results from UED correlate qualitatively with the estimated temperature jump from a saturable absorber model. From this correlation, we conclude that at the high carrier density, the average quantum yield of the nonradiative process approaches unity $(0.87 \pm 0.11)$. Most of the absorbed photon energy converts to lattice vibrations within a picosecond through strong electron-phonon interaction, and this lattice disorder recovers quickly by transporting energy to the substrates on the $\sim 100$-ps time scale. In our study, the maximum displacement prior to damage is only $\sim 5 \%$ of the in-plane lattice constant, which is still lower than the reported theoretical value of $\sim 11.5 \%$ for a phase transition to metallic $\mathrm{MoSe}_{2}{ }^{49}$.

\section{Discussion}

In summary, we have utilized $\mathrm{MeV}$ UED to directly probe lattice dynamics of mono- and bilayer $\mathrm{MoSe}_{2}$. At electron-hole densities in the plasma regime, the nonradiative channel of laser-driven $\mathrm{MoSe}_{2}$ reaches near-unit efficiency with a maximum atomic displacement of $\sim 5 \%$ of the lattice constant. The lattice motions are proportional to the injected carrier density estimated from the reported saturable absorber model. The subpicosecond suppression of Bragg peak intensity and the corresponding disorder of lattice planes are supported by first-principles NAQMD simulations, indicating that the electronic excitation-induced phonon softening is a precursor to efficient dissipation of electronic energy to lattice vibrations. This strong lattice response to optical excitations has important implications for light-induced semiconductor-to-metal structural-phase transition pathways in transition metal dichalcogenides.

\section{Methods}

Sample preparation. Bilayer single-crystal $\mathrm{MoSe}_{2}$ samples were prepared by chemical vapor deposition (CVD) on $\mathrm{Si} / \mathrm{SiO}_{2}$ substrates at $750{ }^{\circ} \mathrm{C}$ and characterized by micro-Raman spectroscopy and photoluminescence measurements (see Supplementary Fig. 6 and Supplementary Note 7 for details), which are consistent with other reported values on different substrates ${ }^{22}$. The $\mathrm{MoSe}_{2}$ samples were transferred onto a ten-nanometer-thick silicon nitride membrane employing a PMMAassisted transfer technique.

Ultrafast electron diffraction. Diffraction experiments were performed at $\mathrm{MeV}$ UED facility at SLAC National Accelerator Laboratory ${ }^{18}$. The pulsed electron beam was operated at a repetition rate of $180 \mathrm{~Hz}$ and an energy of $3.2 \mathrm{MeV}$. The beam with an $\sim 40 \mu \mathrm{m}$ rms spot size impinged on the sample in a normal incidence transmission geometry perpendicular to the (001) plane, therefore probing only inplane lattice displacements ${ }^{29}$. The pulse duration and intensity of the electron beam were $\sim 200 \mathrm{fs}$ and $\sim 20 \mathrm{fC}$, respectively. The $\mathrm{MoSe}_{2}$ bilayers were photoexcited with short optical laser pulses at $400 \mathrm{~nm}(3.1 \mathrm{eV})$ producing hot carriers, and at $800 \mathrm{~nm}(1.55 \mathrm{eV})$ producing band edge carriers. Charge carrier densities shown in all figures were estimated by taking into account the absorption cross section, reflectivity of $\mathrm{MoSe}_{2}$, and interfaces between the sample, PMMA, and silicon nitride membrane on a stack geometry of materials, as shown in Supplementary
Fig. 1. The final absorption was then calibrated to a saturable absorber model, as shown in Supplementary Eq. 1 in Supplementary Note $1^{48}$. The obtained absorption, reflectivity, carrier density, and temperature jump are displayed in Supplementary Tables 1 and 2 .

Nonadiabatic quantum molecular dynamics. NAQMD simulations are performed on a $\mathrm{MoSe}_{2}$ bilayer supercell containing 54 atoms, corresponding to $3 \times 3$ unit cells of the $2-\mathrm{H}$ ground-state crystal structure. Electronic states were calculated using the projector-augmented-wave (PAW) method, and projector functions were generated for $4 d, 5 s$, and $5 p$ states of Mo, and $3 d, 4 s$, and $4 p$ states of Se. The generalized gradient approximation (GGA) was used for the exchange-correlation energy with nonlinear core corrections. The GGA functionals used in this study do not include Hubbard on-site interaction correction. We have performed additional NAQMD simulations to confirm that the inclusion of the on-site interaction term $(U=2 \mathrm{eV})$ does not alter the time constant for lattice thermalization more than 0.01 ps nor the magnitude of atomic vibrations after excitation significantly. Van der Waals interactions were incorporated based on the DFT-D method. Plane-wave cutoff energies were 25 and 250 Ry for electronic pseudo-wave functions and pseudo-charge density, respectively. The energy functional was minimized iteratively using a preconditioned conjugate-gradient method. More details on the implementation of our QMD program can be found in ref. ${ }^{20}$.

Density functional theory. DFT calculations were performed on a $3 \times 3$ supercell of the $2 \mathrm{H}-\mathrm{MoSe}_{2}$ bilayer crystal structure containing 54 atoms. Valence electron wave functions are constructed using a plane-wave basis set containing components up to a kinetic energy of $450 \mathrm{eV}$, and the reciprocal space is sampled using a $7 \times 7 \times 3$ Monkhorst-Pack $\boldsymbol{k}$-point mesh with a Gaussian smearing of orbital occupancies of $0.1 \mathrm{eV}$. The Hessian matrix was generated using density functional perturbation theory, and dispersion relations for normal vibration modes of the $\mathrm{MoSe}_{2}$ unit cell were plotted using the open-source phonopy package ${ }^{50}$. For phonon dispersion calculations, excited states are described within the $\Delta$-SCF DFT framework. In this scheme, a non-Aufbau constraint is imposed on orbital occupancies to promote electrons from the occupied Kohn-Sham energy levels to unoccupied higher-energy levels followed by relaxation of nuclear positions to minima in the potential energy surface defined by the charge density corresponding to the fixed occupancy of these energy levels. The $\Delta$-SCF method is known to provide an inexpensive approximation to the potential energy surface of the excited-state system with accuracy comparable to time-dependent DFT methods ${ }^{51,52}$

Data availability. The data that support the findings of this study are available from the corresponding author upon request.

Received: 31 May 2017 Accepted: 20 October 2017

Published online: 23 November 2017

\section{References}

1. Chakraborty, B. et al. Symmetry-dependent phonon renormalization in monolayer $\mathrm{MoS}_{2}$ transistor. Phys. Rev. B 85, 161403 (2012).

2. Butscher, S., Milde, F., Hirtschulz, M., Malić, E. \& Knorr, A. Hot electron relaxation and phonon dynamics in graphene. Appl. Phys. Lett. 91, 203103 (2007).

3. Wang, H., Zhang, C., Chan, W., Tiwari, S. \& Rana, F. Ultrafast response of monolayer molybdenum disulfide $\left(\mathrm{MoS}_{2}\right)$ photodetectors. Nat. Commun. 6, 17-20 (2015).

4. Schaibley, J. R. et al. Valleytronics in 2D materials. Nat. Rev. Mater. 1, 16055 (2016).

5. Wang, Q. H., Kalantar-Zadeh, K., Kis, A., Coleman, J. N. \& Strano, M. S. Electronics and optoelectronics of two-dimensional transition metal dichalcogenides. Nat. Nanotechnol. 7, 699-712 (2012).

6. Larentis, S., Fallahazad, B. \& Tutuc, E. Field-effect transistors and intrinsic mobility in ultra-thin $\mathrm{MoSe}_{2}$ layers. Appl. Phys. Lett. 101, 223104 (2012).

7. Kumar, N. et al. Exciton-exciton annihilation in $\mathrm{MoSe}_{2}$ monolayers. Phys. Rev B 89, 125427 (2014).

8. Sun, D. et al. Observation of rapid exciton-exciton annihilation in monolayer molybdenum disulfide. Nano Lett. 14, 5625-5629 (2014).

9. Yu, Y. et al. Fundamental limits of exciton-exciton annihilation for light emission in transition metal dichalcogenide monolayers. Phys. Rev. B 93, 201111 (2016).

10. Wang, H., Zhang, C. \& Rana, F. Ultrafast dynamics of defect-assisted electronhole recombination in monolayer $\mathrm{MoS}_{2}$. Nano Lett. 15, 339-345 (2015).

11. Wang, H. et al. Fast exciton annihilation by capture of electrons or holes by defects via Auger scattering in monolayer metal dichalcogenides. Phys. Rev. B 91, 165411 (2015). 
12. Mak, K. F., Lee, C., Hone, J., Shan, J. \& Heinz, T. F. Atomically thin $\mathrm{MoS}_{2}$ : a new direct-gap semiconductor. Phys. Rev. Lett. 105, 136805 (2010).

13. Defo, R. K. et al. Strain dependence of band gaps and exciton energies in pure and mixed transition-metal dichalcogenides. Phys. Rev. B 94, 155310 (2016).

14. Shen, T., Penumatcha, A. V. \& Appenzeller, J. Strain engineering for transition metal dichalcogenides based field effect transistors. ACS Nano 10, 4712-4718 (2016).

15. Amani, M. et al. Near-unity photoluminescence quantum yield in $\mathrm{MoS}_{2}$. Science 350, 1065-1068 (2015).

16. Han, H.-V. et al. Photoluminescence enhancement and structure repairing of monolayer $\mathrm{MoSe}_{2}$ by hydrohalic acid treatment. ACS Nano 10, 1454-1461 (2016).

17. Ruppert, C., Chernikov, A., Hill, H. M., Rigosi, A. F. \& Heinz, T. F. The role of electronic and phononic excitation in the optical response of monolayer $\mathrm{WS}_{2}$ after ultrafast excitation. Nano Lett. 17, 644-651 (2017).

18. Weathersby, S. P. et al. Mega-electron-volt ultrafast electron diffraction at SLAC national accelerator laboratory. Rev. Sci. Instrum. 86, 73702 (2015).

19. Shimamura, K. et al. Hydrogen-on-demand using metallic alloy nanoparticles in water. Nano Lett. 14, 4090-4096 (2014).

20. Shimojo, F. et al. A divide-conquer-recombine algorithmic paradigm for large spatiotemporal quantum molecular dynamics simulations. J. Chem. Phys. 140, 18A529 (2014).

21. Meng, S. \& Kaxiras, E. Mechanisms for ultrafast nonradiative relaxation in electronically excited eumelanin constituents. Biophys. J. 95, 4396-4402 (2008).

22. Ugeda, M. M. et al. Giant bandgap renormalization and excitonic effects in a monolayer transition metal dichalcogenide semiconductor. Nat. Mater. 13, 1091-1095 (2014).

23. Mannebach, E. M. et al. Dynamic structural response and deformations of monolayer $\mathrm{MoS}_{2}$ visualized by femtosecond electron diffraction. Nano. Lett. 15, 6889-6895 (2015).

24. Steinhoff, A., Rosner, M., Jahnke, F., Wehling, T. O. \& Gies, C. Influence of excited carriers on the optical and electronic properties of $\mathrm{MoS}_{2}$. Nano Lett. 14, 3743-3748 (2014)

25. Chernikov, A., Ruppert, C., Hill, H. M., Rigosi, A. F. \& Heinz, T. F. Population inversion and giant bandgap renormalization in atomically thin $\mathrm{WS}_{2}$ layers. Nat. Photonics 9, 466-470 (2015).

26. Nie, Z. et al. Ultrafast carrier thermalization and cooling dynamics in FewLayer $\mathrm{MoS}_{2}$. ACS Nano 8, 10931-10940 (2014).

27. Qiu, D. Y., da Jornada, F. H. \& Louie, S. G. Screening and many-body effects in two-dimensional crystals: Monolayer $\mathrm{MoS}_{2}$. Phys. Rev. B 93, 235435 (2016).

28. Wang, H., Zhang, C. \& Rana, F. Surface recombination limited lifetimes of photoexcited carriers in Few-Layer transition metal dichalcogenide $\mathrm{MoS}_{2}$. Nano Lett. 15, 8204-8210 (2015).

29. Chatelain, R. P., Morrison, V. R., Klarenaar, B. L. M. \& Siwick, B. J. Coherent and incoherent electron-phonon coupling in graphite observed with radiofrequency compressed ultrafast electron diffraction. Phys. Rev. Lett. 113, 235502 (2014).

30. Harb, M. et al. Phonon-phonon interactions in photoexcited graphite studied by ultrafast electron diffraction. Phys. Rev. B 93, 104104 (2016).

31. Lundberg, M. The crystal structure of $\mathrm{MoSe}_{2}$. Acta. Crystallogr. 16, 1183 (1963).

32. Sokolowski-Tinten, K. et al. Femtosecond X-ray measurement of coherent lattice vibrations near the Lindemann stability limit. Nature 422, 287-289 (2003).

33. Lindenberg, A. M. et al. Atomic-Scale visualization of inertial dynamics. Science 308, 392-395 (2005)

34. Gaffney, K. J. et al. Observation of structural anisotropy and the onset of liquidlike motion during the nonthermal melting of InSb. Phys. Rev. Lett. 95, 125701 (2005).

35. Sciaini, G. et al. Electronic acceleration of atomic motions and disordering in bismuth. Nature 458, 56-59 (2009).

36. Bader, S. D., Sinha, S. K., Schweiss, B. P. \& Renker, B. in Superconductivity in Ternary Compounds I: 223-239 (Springer-Verlag, Berlin Heidelberg, 1982).

37. Keum, D. H. et al. Bandgap opening in few-layered monoclinic $\mathrm{MoTe}_{2}$. Nat. Phys. 11, 482-486 (2015).

38. Kolobov, A. V., Fons, P. \& Tominaga, J. Electronic excitation-induced semiconductor-to-metal transition in monolayer $\mathrm{MoTe}_{2}$. Phys. Rev. B 94, 94114 (2016).

39. Allen, P. B. \& Cohen, M. L. Superconductivity and phonon softening. Phys. Rev. Lett. 29, 1593-1596 (1972).

40. Moussa, J. E. \& Cohen, M. L. Two bounds on the maximum phonon-mediated superconducting transition temperature. Phys. Rev. B 74, 94520 (2006).

41. Zeng, S., Zhao, Y., Li, G. \& Ni, J. Strongly enhanced superconductivity in doped monolayer $\mathrm{MoS}_{2}$ by strain. Phys. Rev. B 94, 24501 (2016).

42. Poole, C., Farach, H. \& Creswick, R. Handbook of Superconductivity. Ch 9 (Academic Press, 2000).
43. Cadiz, F. et al. Ultralow power threshold for laser induced changes in optical properties of 2D Molybdenum dichalcogenides. 2D Mater. 3, 45008 (2016).

44. Als-Nielsen, J. \& McMorrow, D. Elements of Modern X-ray Physics: 2nd Edn. (WILEY, Chichester, 2011).

45. Liu, Y. et al. Thermally driven (Mo, $\mathrm{W})-\left(\mathrm{S}_{2}, \mathrm{Se}_{2}\right)$ phonon and photon energy relaxation dynamics. J. Phys. Chem. C 119, 25071-25076 (2015).

46. Hardy, K. A., Parker, F. T. \& Walker, J. C. A better approximation to the Debye-Waller factor. Nucl. Instrum. Methods 86, 171-172 (1970).

47. Kiwia, H. L. \& Westrum, E. F. Low-temperature heat capacities of molybdenum diselenide and ditelluride. J. Chem. Thermodyn. 7, 683-691 (1975).

48. Wang, K. et al. Broadband ultrafast nonlinear absorption and nonlinear refraction of layered molybdenum dichalcogenide semiconductors. Nanoscale 6, 10530-10535 (2014).

49. Yun, W. S., Han, S. W., Hong, S. C., Kim, I. G. \& Lee, J. D. Thickness and strain effects on electronic structures of transition metal dichalcogenides: $2 \mathrm{H}-\mathrm{MX}_{2}$ semiconductors (M = Mo,W; X=S,Se,Te). Phys. Rev. B 85, 33305 (2012).

50. Togo, A. \& Tanaka, I. First principles phonon calculations in materials science. Scr. Mater. 108, 1-5 (2015).

51. Gavnholt, J., Olsen, T., Engelund, M. \& Schiøtz, J. $\Delta$ self-consistent field method to obtain potential energy surfaces of excited molecules on surfaces. Phys. Rev. B 78, 75441 (2008).

52. Kowalczyk, T., Yost, S. R. \& Van Voorhis, T. Assessment of the $\Delta$ SCF density functional theory approach for electronic excitations in organic dyes. J. Chem. Phys. 134, 54128 (2011).

\section{Acknowledgements}

This work was supported as part of the Computational Materials Sciences Program funded by the U.S. Department of Energy, Office of Science, and Basic Energy Sciences, under Award Number DE-SC00014607. The UED work was performed at SLAC MeVUED, which is supported in part by the DOE BES SUF Division Accelerator \& Detector R\&D program, the LCLS Facility, and SLAC under contract Nos. DE-AC02-05-CH11231 and DE-AC02-76SF00515. Simulations were performed at the Argonne Leadership Computing Facility under the DOE INCITE program at the Center for High Performance Computing of the University of Southern California and the Extreme Science and Engineering Discovery Environment (XSEDE), which is supported by National Science Foundation grant number ACI-1548562. The authors would like to thank Prof. Aaron Lindenberg and Dr. Ehern Mannebach for their valuable discussions of data analysis in electron diffraction.

\section{Author contributions}

A.N., D.M.F., P.A., P.V., R.K. and U.B.: Designed the research. V.K., X.Z., A.A., C.S.T. and P.A.: Synthesized and characterized the experimental samples. C.W., M.-F.L., Q.Z. R.L. and X.W.: Performed the UED experiment. A.K., A.N., F.S., L.B., P.V. and R.K. Performed ab initio calculations. All authors contributed to the analysis, discussion, and preparation of the manuscript.

\section{Additional information}

Supplementary Information accompanies this paper at doi:10.1038/s41467-017-01844-2.

Competing interests: The authors declare no competing financial interests.

Reprints and permission information is available online at http://npg.nature.com/ reprintsandpermissions/

Publisher's note: Springer Nature remains neutral with regard to jurisdictional claims in published maps and institutional affiliations.

Open Access This article is licensed under a Creative Commons Attribution 4.0 International License, which permits use, sharing, adaptation, distribution and reproduction in any medium or format, as long as you give appropriate credit to the original author(s) and the source, provide a link to the Creative Commons license, and indicate if changes were made. The images or other third party material in this article are included in the article's Creative Commons license, unless indicated otherwise in a credit line to the material. If material is not included in the article's Creative Commons license and your intended use is not permitted by statutory regulation or exceeds the permitted use, you will need to obtain permission directly from the copyright holder. To view a copy of this license, visit http://creativecommons.org/ licenses/by/4.0/.

(C) The Author(s) 2017 